\title{
Thermodynamics of two aligned Kerr-Newman black holes
}

\author{
H. García-Compeán, V. S. Manko and C. J. Ramírez-Valdez \\ ${ }^{\dagger}$ Departamento de Física, Centro de Investigación y de Estudios Avanzados del IPN, \\ A.P. 14-740, 07000 Ciudad de México, Mexico
}

\begin{abstract}
Using the notion of thermodynamic length, the first law of thermodynamics is consistently derived for two binary configurations of equal Kerr-Newman black holes separated by a massless strut. Like in the electrostatic systems of two Reissner-Nordström black holes and stationary vacuum systems of two Kerr black holes considered earlier, the thermodynamic length $\ell$ turns out to be defined by the same simple formula $\ell=L \exp \left(\gamma_{0}\right), L$ being the coordinate length of the strut and $\gamma_{0}$ the value of the metric function $\gamma$ on the strut, which permits the elaboration of $\ell$ in a concise analytic form. The expression of the free energy in the case of two generic Kerr-Newman black holes is also proposed.
\end{abstract}

PACS numbers: 04.20.Jb, 04.70.Bw, 97.60.Lf 


\section{INTRODUCTION}

In our previous paper [1] we have performed analytical derivation of the first law of thermodynamics for the binary configurations of Kerr black holes [2] with the aid of the notion of thermodynamic length introduced by Appels, Gregory and Kubizñák [3], and we have shown that in the stationary vacuum case the thermodynamic length is defined by the same formula as in the electrostatic case analyzed in [4]. It was also suggested in [1] that thermodynamics of the Kerr-Newman (KN) black holes [5] in binary systems could probably be treated as readily as in the aforementioned two cases, and the aim of the present paper is to demonstrate that this is indeed true when the usual approach to the thermodynamic length is employed in the derivation procedures.

The binary configurations of our interest are obtainable from the double-Kerr-Newman solution [6] by imposing certain conditions on the metric functions. Since the latter conditions have not yet been solved analytically in the general case of two black-hole constituents separated by a strut [7], our consideration will be restricted to two different configurations of equal counterrotating $\mathrm{KN}$ black holes, in one of which the charges of the constituents have the same signs and in the other the charges have opposite signs. The first configuration is described by the Bretón-Manko (BM) electrovac solution [8] for which a physical parametrization was worked out in [9]; on the other hand, the binary system with opposite charges is described by a 4-parameter specialization of the Ernst-Manko-Ruiz equatorially antisymmetric solution [10] whose physical parametrization was worked out in [11]. Actually, both the above electrovac solutions may be considered as two different charging generalizations of the vacuum BM spacetime studied in our previous paper [1]. Mention also that one of the virtues of the solutions for equal counterrotating KN black holes is that the rotation parameter entering these solutions represents exactly the angular momentum per unit mass of each black-hole constituent, which simplifies considerably the related symbolic computations.

Our paper is organized as follows. In Sec. II we derive the first law of thermodynamics for the binary system of KN black holes described by the BM solution. Here we also give the form of the Euclidean action for this system and show that the corresponding free energy involving explicitly the thermodynamic length is able to reproduce exactly the expressions of the entropy and interaction force. In Sec. III the analogous thermodynamic analysis 
is performed for the binary configuration of counterrotating KN black holes endowed with opposite charges, and it confirms the main conclusions of the previous section. The discussion of the results obtained, with possible extensions to the binary systems of unequal KN black holes can be found in Sec. IV. Concluding remarks are given in Sec. V.

\section{TWO EQUAL COUNTERROTATING KN BLACK HOLES CARRYING THE SAME CHARGES}

The BM 4-parameter electrovac solution for two identical counterrotating KN black holes is described by the line element

$$
d s^{2}=f^{-1}\left[e^{2 \gamma}\left(d \rho^{2}+d z^{2}\right)+\rho^{2} d \varphi^{2}\right]-f(d t-\omega d \varphi)^{2}
$$

with the metric functions $f, \gamma$ and $\omega$ of the form [9]

$$
\begin{aligned}
f= & \frac{A \bar{A}-B \bar{B}+C \bar{C}}{(A+B)(\bar{A}+\bar{B})}, \quad e^{2 \gamma}=\frac{A \bar{A}-B \bar{B}+C \bar{C}}{16 R^{4} \sigma^{4} R_{+} R_{-} r_{+} r_{-}}, \quad \omega=-\frac{\operatorname{Im}[2 G(\bar{A}+\bar{B})+C \bar{I}]}{A \bar{A}-B \bar{B}+C \bar{C}}, \\
A= & \left(M^{2}-Q^{2}\right)\left[4 \sigma^{2}\left(R_{+} R_{-}+r_{+} r_{-}\right)+R^{2}\left(R_{+} r_{+}+R_{-} r_{-}\right)\right]+\left[\sigma^{2}\left(R^{2}-4 M^{2}+4 Q^{2}\right)\right. \\
& \left.-J^{2} R^{2} \mu\right]\left(R_{+} r_{-}+R_{-} r_{+}\right)-2 i J R \sigma \mu\left(M R+2 M^{2}-Q^{2}\right)\left(R_{+} r_{-}-R_{-} r_{+}\right), \\
B= & 2 M R \sigma\left[R \sigma\left(R_{+}+R_{-}+r_{+}+r_{-}\right)-\lambda\left(R_{+}-R_{-}-r_{+}+r_{-}\right)\right], \\
C= & Q B / M, \\
G= & -z B+R \sigma\left\{\left(2 M^{2}-Q^{2}\right)\left[2 \sigma\left(r_{+} r_{-}-R_{+} R_{-}\right)+R\left(R_{-} r_{-}-R_{+} r_{+}\right)\right]\right. \\
& \left.+M(R+2 \sigma)(R \sigma-\lambda)\left(R_{+}-r_{-}\right)+M(R-2 \sigma)(R \sigma+\lambda)\left(R_{-}-r_{+}\right)\right\}, \\
I= & \frac{Q}{M}\left\{G+R Q^{2} \sigma\left[2 \sigma\left(r_{+} r_{-}-R_{+} R_{-}\right)+R\left(R_{-} r_{-}-R_{+} r_{+}\right)\right]\right\}, \\
\lambda \equiv & 2\left(M^{2}-Q^{2}\right)+i J \mu\left(M R+2 M^{2}-Q^{2}\right), \\
R_{ \pm}= & \sqrt{\rho^{2}+\left(z+\frac{1}{2} R \pm \sigma\right)^{2}}, \quad r_{ \pm}=\sqrt{\rho^{2}+\left(z-\frac{1}{2} R \pm \sigma\right)^{2}},
\end{aligned}
$$

where the arbitrary real parameters $M$ and $Q$ are, respectively, the mass and electric charge of each black hole, while $J$ is the angular momentum of the lower black hole (the angular momentum of the upper black hole is $-J) ; R$ denotes the coordinate distance between the

centers of black holes (see Fig. 1). The constant quantity $\sigma$ determining horizon's length is defined by the expression

$$
\sigma=\sqrt{M^{2}-Q^{2}-J^{2} \mu}, \quad \mu \equiv \frac{R^{2}-4 M^{2}+4 Q^{2}}{\left(M R+2 M^{2}-Q^{2}\right)^{2}} .
$$


Besides, the $t$ and $\varphi$ components of the electromagnetic 4-potential are given by the formulas

$$
A_{t}=-\operatorname{Re}\left(\frac{C}{A+B}\right), \quad A_{\varphi}=\operatorname{Im}\left(\frac{I}{A+B}\right) .
$$

Since we are interested exclusively in the black-hole sector of the BM solution, then $\sigma$ must be a real quantity, which means that the parameters of the solution are subject to the constraint $\sigma^{2}>0$. Note that in the above formulas we have introduced explicitly the angular momentum $J$ (of the lower black hole) instead of the parameter $a$ used in [11], which is more suitable for the analysis of thermodynamics of the BM solution. In the absence of electric charge $(Q=0)$, one recovers from (11)-(3) the vacuum solution for two counterrotating Kerr black holes considered in our previous paper, and in Appendix we give its representation obtainable from the general formulas for binary configurations of nonequal black holes that might represent interest for applications.

Each black hole of the BM solution satisfies Smarr's mass formula [12]

$$
M=2 T S+2 \Omega J+\Phi_{e} Q
$$

where $T$ is the temperature and $S$ the entropy of each black hole, $\Omega$ is horizon's angular velocity of the lower black hole ( $-\Omega$ of the upper black hole) and $\Phi_{e}$ is the electric potential. For the quantities $S, \Omega$ and $\Phi_{e}$, as well as for the interaction force $\mathcal{F}$, the paper [9] gives the following expressions:

$$
\begin{aligned}
T & =\frac{R \sigma}{2 \pi \Delta}, \quad S=\frac{\pi \Delta}{R}, \quad \Omega=\frac{J \mu\left(M R+2 M^{2}-Q^{2}\right)}{\Delta}, \\
\Phi_{e} & =\frac{Q\left[(R+2 M)(M+\sigma)-2 Q^{2}\right]}{\Delta}, \quad \mathcal{F}=\frac{M^{2}-Q^{2}}{R^{2}-4 M^{2}+4 Q^{2}}, \\
\Delta & \equiv 2 M(R+2 M)(M+\sigma)-Q^{2}(R+4 M+2 \sigma) .
\end{aligned}
$$

To derive consistently the first law of thermodynamics and find the explicit form of the corresponding thermodynamic length $\ell$ for this binary configuration, we must take differentials of the four quantities $S, \Omega, \Phi_{e}$ and $\mathcal{F}$ by considering these as functions of $M, J, R$ and $Q$, and solve the resulting system for $d M, d \Omega, d \Phi_{e}$ and $d R$, of which for the differential $d M$ we get the expression

$$
d M=T d S+\Omega d J+\Phi_{e} d Q-\frac{1}{2 R^{2}}(R-2 \sigma)\left(R^{2}-4 M^{2}+4 Q^{2}\right) d \mathcal{F} .
$$

Taking into account the equality of black holes, we finally arrive at the desired first law and thermodynamic length in the form

$$
d M_{T}=2 T d S+2 \Omega d J+2 \Phi_{e} d Q-\ell d \mathcal{F}
$$




$$
M_{T}=2 M, \quad \ell=(R-2 \sigma)\left(1-\frac{4\left(M^{2}-Q^{2}\right)}{R^{2}}\right),
$$

and it is not difficult to show using formulas (2)-(3) that, similar to the electrostatic case considered in [4] or the pure vacuum case considered in [1], the thermodynamic length $\ell$ is defined by the formula

$$
\ell=L e^{\gamma_{0}}
$$

where $L=R-2 \sigma$ is the coordinate length of the strut (see Fig. 1), and $\gamma_{0}$ is the value of the metric function $\gamma$ on the strut. It might be tempting to think looking at (8) that $\ell=L$ when $M^{2}=Q^{2}$; however, in this particular case the conical singularity disappears (since $\mathcal{F}=0$ ) and the two constituents are in neutral equilibrium independent of the separation distance due to balance of the gravitational and electromagnetic forces: for nonvanishing angular momentum the resulting equilibrium configuration is described by the Parker-Ruffini-Wilkins metric [13] for hyperextreme KN sources, while for vanishing angular momentum the equilibrium is between two extreme charged black holes [14, 15].

To gain a better insight into thermodynamics of the BM binary configuration, we have computed, via standard procedures, the Euclidean action [16] corresponding to this configuration, yielding the simple expression

$$
I=\beta\left(M-\Phi_{e} Q+\ell \mathcal{F}\right)
$$

where $\beta=1 / T$, and the quantities $\Phi_{e}, \mathcal{F}$ and $\ell$ are defined by the formulas (6) and (7). Hence, taking into account that $I=\beta W, W$ being the free energy, we get

$$
W=M-\Phi_{e} Q+\ell \mathcal{F}
$$

To find the variation of the thermodynamic potential $W$, we must first take differentials of the quantities $\ell, T, \Omega$ and $\Phi_{e}$ by considering these as functions of the parameters $M, J$, $Q$ and $R$ and then express $d M, d J, d Q$ and $d R$ in terms of the differentials $d \ell, d T, d \Omega$ and $d \Phi_{e}$ by solving the linear system of four algebraic equations. Expressing subsequently the differential $d W$ in terms of the latter four differentials, we finally arrive at the formula

$$
d W=-2 S d T-2 J d \Omega-2 Q d \Phi_{e}+\mathcal{F} d \ell
$$

whence it follows that

$$
S=-\left.\frac{1}{2} \frac{\partial W}{\partial T}\right|_{\Omega, \Phi_{e}, \ell}, \quad J=-\left.\frac{1}{2} \frac{\partial W}{\partial \Omega}\right|_{T, \Phi_{e}, \ell}, \quad Q=-\left.\frac{1}{2} \frac{\partial W}{\partial \Phi_{e}}\right|_{T, \Omega, \ell}, \quad \mathcal{F}=\left.\frac{\partial W}{\partial \ell}\right|_{T, \Omega, \Phi_{e}} .
$$


It is remarkable that the relations (12) and (13) are verified exactly due to the use in (11) of the thermodynamic length $\ell$. We also note that derivation of the formula (12) is computationally a more complicated task than obtaining the first law of thermodynamics in the form (8) .

\section{TWO EQUAL COUNTERROTATING KN BLACK HOLES WITH OPPOSITE CHARGES}

While the BM solution dates back to 1995, the binary configuration of equal KN counterrotating black holes with opposite electric charges was identified and correctly described only quite recently [11]. It is determined by the metric functions of the form

$$
\begin{aligned}
& f=\frac{A \bar{A}-B \bar{B}+C \bar{C}}{(A+B)(\bar{A}+\bar{B})}, \quad e^{2 \gamma}=\frac{A \bar{A}-B \bar{B}+C \bar{C}}{16 R^{4} \sigma^{4} R_{+} R_{-} r_{+} r_{-}}, \quad \omega=-\frac{\operatorname{Im}[2 G(\bar{A}+\bar{B})+C \bar{I}]}{A \bar{A}-B \bar{B}+C \bar{C}}, \\
& A=R^{2}\left(M^{2}-Q^{2} \nu\right)\left(R_{+}-R_{-}\right)\left(r_{+}-r_{-}\right)+4 \sigma^{2}\left(M^{2}+Q^{2} \nu\right)\left(R_{+}-r_{+}\right)\left(R_{-}-r_{-}\right) \\
& +2 R \sigma\left[R \sigma\left(R_{+} r_{-}+R_{-} r_{+}\right)+i J \mu\left(R_{+} r_{-}-R_{-} r_{+}\right)\right] \\
& B=2 M R \sigma\left[R \sigma\left(R_{+}+R_{-}+r_{+}+r_{-}\right)-\left(2 M^{2}-i J \mu\right)\left(R_{+}-R_{-}-r_{+}+r_{-}\right)\right] \text {, } \\
& C=2 C_{0} R \sigma\left[(R+2 \sigma)\left(R \sigma-2 M^{2}-i J \mu\right)\left(r_{+}-R_{-}\right)+(R-2 \sigma)\right. \\
& \left.\times\left(R \sigma+2 M^{2}+i J \mu\right)\left(r_{-}-R_{+}\right)\right], \\
& G=-z B+R \sigma\left\{R\left(2 M^{2}-Q^{2} \nu\right)\left(R_{-} r_{-}-R_{+} r_{+}\right)+2 \sigma\left(2 M^{2}+Q^{2} \nu\right)\left(r_{+} r_{-}-R_{+} R_{-}\right)\right. \\
& +M\left[(R+2 \sigma)\left(R \sigma-2 M^{2}+i J \mu\right)+2(R-2 \sigma) Q^{2} \nu\right]\left(R_{+}-r_{-}\right) \\
& \left.+M\left[(R-2 \sigma)\left(R \sigma+2 M^{2}-i J \mu\right)-2(R+2 \sigma) Q^{2} \nu\right]\left(R_{-}-r_{+}\right)\right\}, \\
& I=-z C+2 C_{0} M\left[R^{2}\left(2 M^{2}-2 \sigma^{2}+i J \mu\right)\left(R_{+} r_{+}+R_{-} r_{-}\right)\right. \\
& \left.+2 \sigma^{2}\left(R^{2}-4 M^{2}-2 i J \mu\right)\left(R_{+} R_{-}+r_{+} r_{-}\right)\right]-C_{0}\left(R^{2}-4 \sigma^{2}\right) \\
& \times\left\{2 M\left[R \sigma\left(R_{+} r_{-}-R_{-} r_{+}\right)+\left(2 M^{2}+i J \mu\right)\left(R_{+} r_{-}+R_{-} r_{+}\right)\right]+R \sigma[R \sigma\right. \\
& \left.\left.\times\left(R_{+}+R_{-}+r_{+}+r_{-}\right)+\left(6 M^{2}+i J \mu\right)\left(R_{+}-R_{-}-r_{+}+r_{-}\right)+8 M R \sigma\right]\right\}, \\
& R_{ \pm}=\sqrt{\rho^{2}+\left(z+\frac{1}{2} R \pm \sigma\right)^{2}}, \quad r_{ \pm}=\sqrt{\rho^{2}+\left(z-\frac{1}{2} R \pm \sigma\right)^{2}},
\end{aligned}
$$

where the dimensionless quantities $\mu, \nu$ and $C_{0}$ are defined as

$$
\mu=\frac{R^{2}-4 M^{2}}{M(R+2 M)+Q^{2}}, \quad \nu=\frac{R^{2}-4 M^{2}}{(R+2 M)^{2}+4 Q^{2}}, \quad C_{0}=-\frac{Q\left(R^{2}-4 M^{2}+2 i J \mu\right)}{(R+2 M)\left(R^{2}-4 \sigma^{2}\right)},
$$


and the horizons' half length $\sigma$ is given by the expression

$$
\sigma=\sqrt{M^{2}-\left(\frac{J^{2}\left[(R+2 M)^{2}+4 Q^{2}\right]}{\left[M(R+2 M)+Q^{2}\right]^{2}}+Q^{2}\right) \frac{R-2 M}{R+2 M}} .
$$

The arbitrary parameters of the solution are $M, J, Q$ and $R$ which have the same meaning as in the previous section, with the only reservation that now $J$ is the angular momentum of the upper constituent ( $-J$ of the lower constituent, whose electric charge is $-Q$, see Fig. 2). Note that in the above formulas $J$ has been introduced explicitly as the rotation parameter instead of the parameter $a$ used in [11]. The electromagnetic field is defined by Eqs. (4) of the previous section. The black-hole sector of the solution (14)-(16), as usual, corresponds to $\sigma^{2}>0$. One can see that the formulas describing the case of opposite charges are somewhat more complicated than those of the case of identical charges.

The two KN black holes have the same temperature $T$ and entropy $S$, but their angular momenta, charges, horizon's angular velocities and electric potentials have opposite signs; nonetheless, the Smarr formula (5) is verified by each black hole, and below we give the form of $T, S, \Omega, \Phi_{e}$ obtained in [11] for the upper constituent, to which the expression of the interaction force $\mathcal{F}$ is also added:

$$
\begin{aligned}
T & =\frac{R \sigma\left[(R+2 M)^{2}+4 Q^{2}\right]}{2 \pi(R+2 M)^{2}\left[2(M+\sigma)\left(M R+2 M^{2}+Q^{2}\right)-Q^{2}(R-2 M)\right]}, \\
S & =\frac{\pi}{R(R+2 \sigma)}\left((R+2 M)^{2}(M+\sigma)^{2}+\frac{J^{2}\left(R^{2}-4 M^{2}\right)^{2}}{\left(M R+2 M^{2}+Q^{2}\right)^{2}}\right), \\
\Omega & =\frac{J\left[2(M-\sigma)\left(M R+2 M^{2}+Q^{2}\right)-Q^{2}(R-2 M)\right]}{\left(4 J^{2}+Q^{4}\right)\left(M R+2 M^{2}+Q^{2}\right)}, \\
\Phi_{e} & =\frac{Q\left[Q^{2}(M-\sigma)\left(M R+2 M^{2}+Q^{2}\right)+2 J^{2}(R-2 M)\right]}{\left(4 J^{2}+Q^{4}\right)\left(M R+2 M^{2}+Q^{2}\right)}, \\
\mathcal{F} & =\frac{M^{2}(R+2 M)^{2}+Q^{2} R^{2}}{(R+2 M)^{2}\left(R^{2}-4 M^{2}\right)} .
\end{aligned}
$$

The procedure of obtaining the first law of thermodynamics for this second binary configuration involving the quantities $S, \Omega, \Phi_{e}$ and $\mathcal{F}$ is fully analogous to the already considered in the previous section during the derivation of equations (8), so we will restrict ourselves to just writing down the final result for the first law and corresponding thermodynamic length:

$$
\begin{aligned}
d M_{T} & =2 T d S+2 \Omega d J+2 \Phi_{e} d Q-\ell d \mathcal{F}, \\
M_{T} & =2 M, \quad \ell=\frac{(R-2 \sigma)(R+2 M)^{2}\left(R^{2}-4 M^{2}\right)}{R^{2}\left[(R+2 M)^{2}+4 Q^{2}\right]} .
\end{aligned}
$$


A direct check shows that the above $\ell$ verifies formula (9).

A thorough straightforward analysis of the Euclidean action and free energy of the solution reveals that these have exactly the same structure defined by formulas (10) and (11) as in the case of the BM configuration, including the formula (12) for the variation of $W$. Therefore, we have got another evidence that the thermodynamic length determines in a natural and consistent way the thermodynamical properties of the binary systems of KN black holes.

\section{DISCUSSION}

While the case of equal black holes is very suitable for the thermodynamic analysis because the binary configurations are in thermal equilibrium allowing for the introduction of the free energy potential through the Euclidean action, the question still arises of how such a potential could be defined when a binary configuration is composed of unequal black holes having different temperatures. We recall in this respect that even in the simplest case of the double-Schwarzschild solution [17] the paper [18] for instance does not analyze the general case of two Schwarzschild black holes, most probably because of the difficulties in the description of the Euclidean action when a binary system is not in thermal equilibrium. Nonetheless it is remarkable that, thanks to the thermodynamic length, the free energy $W$ in the case of nonequal black-hole constituents can be easily guessed from the expressions of $W$ obtained for various binary configurations of equal constituents. Indeed, in one assumes that the contribution of the strut in $W$ is defined by the term $\ell \mathcal{F}$, then the expression for $W$ in the general double-Schwarzschild solution takes the form $\left(m_{i}\right.$ are the masses of black holes and $R$ the distance between the black hole centers)

$$
\begin{aligned}
W & =\frac{1}{2}\left(m_{1}+m_{2}\right)+\ell \mathcal{F} \\
\ell & =\left(R-m_{1}-m_{2}\right) \frac{R^{2}-\left(m_{1}+m_{2}\right)^{2}}{R^{2}-\left(m_{1}-m_{2}\right)^{2}}, \quad \mathcal{F}=\frac{m_{1} m_{2}}{R^{2}-\left(m_{1}+m_{2}\right)^{2}},
\end{aligned}
$$

where the above expression for $\ell$ was found in [4]. Then, using formulas for the temperatures $T_{1}$ and $T_{2}$ of the black holes from [4] and the expression of $\ell$ from (19) we shall get, via the procedure described in Sec. II, that

$$
d W=-S_{1} d T_{1}-S_{2} d T_{2}+\mathcal{F} d \ell
$$




$$
S_{1}=\frac{4 \pi m_{1}\left(R+m_{1}+m_{2}\right)}{R+m_{1}-m_{2}}, \quad S_{2}=\frac{4 \pi m_{2}\left(R+m_{1}+m_{2}\right)}{R-m_{1}+m_{2}},
$$

$S_{1}$ and $S_{2}$ being the entropies of the Schwarzschild black holes, so that

$$
S_{1}=-\left.\frac{\partial W}{\partial T_{1}}\right|_{T_{2}, \ell}, \quad S_{2}=-\left.\frac{\partial W}{\partial T_{2}}\right|_{T_{1}, \ell} .
$$

Note that for the derivation of (20) it is not sufficient only to calculate correctly the contribution of the strut in (19), namely,

$$
\frac{m_{1} m_{2}\left(R-m_{1}-m_{2}\right)}{R^{2}-\left(m_{1}-m_{2}\right)^{2}}
$$

but it is of paramount importance to present the quantity (22) in the form $\ell \mathcal{F}$, because any other presentation of (22), say, in the form $L F$, with $L=R-m_{1}-m_{2}$ and $F=$ $m_{1} m_{2} /\left[R^{2}-\left(m_{1}-m_{2}\right)^{2}\right]$, will not permit to derive the correct expression for $d W$ leading to (21) exactly (even in the case of equal constituents).

Moreover, we have also investigated the issue of the free energy potential for all the binary configurations of Kerr black holes considered in our previous paper [1], as well as for the double-Reissner-Nordström solution [19, 20] analyzed in [4], and this endeavor has made us tentatively conclude that the expression of the free energy in the binary system of generic KN black holes separated by a massless strut and described by seven independent parameters $M_{i}, J_{i}, Q_{i}$ and $\mathrm{R}, i=1,2$, must have the form

$$
W=\frac{1}{2} \sum_{i=1}^{2}\left(M_{i}-Q_{i} \Phi_{i}\right)+\ell \mathcal{F}
$$

while the variation of $W$ is given by the formula

$$
d W=-\sum_{i=1}^{2}\left(S_{i} d T_{i}+J_{i} d \Omega_{i}+Q_{i} d \Phi_{i}\right)+\mathcal{F} d \ell,
$$

where $S_{i}, T_{i}, \Omega_{i}$ and $\Phi_{i}$ stand, respectively, for the individual entropies, temperatures, horizon's angular velocities and electric potentials of the black holes (of course, $M_{i}, J_{i}$ and $Q_{i}$ are the masses, angular momenta and electric charges of black holes). Besides, the corresponding first law of thermodynamics reads as

$$
d\left(M_{1}+M_{2}\right)=\sum_{i=1}^{2}\left(T_{i} d S_{i}+\Omega_{i} d J_{i}+\Phi_{i} d Q_{i}\right)-\ell d \mathcal{F} .
$$

The thermodynamic length $\ell$ entering (23) $-(25)$ is universally defined by the formula (9) first obtained in [4], and its geometrical interpretation proposed in the latter paper is the area of the worldsheet of the strut per unit time. 


\section{APPENDIX}

Here we give a possible concise representation of the vacuum BM solution worked out

with the aid of the general formulas of the paper [1]. Its Ernst potential [21] is determined by the formulas

$$
\begin{aligned}
\mathcal{E} & =(A-B) /(A+B), \\
A & =R^{2}\left(R_{+}-R_{-}\right)\left(r_{+}-r_{-}\right)-4 \sigma^{2}\left(R_{+}-r_{+}\right)\left(R_{-}-r_{-}\right), \\
B & =2 R \sigma\left[(R+2 \sigma)\left(R_{-}-r_{+}\right)-(R-2 \sigma)\left(R_{+}-r_{-}\right)\right] \\
r_{ \pm} & =\frac{ \pm \sigma-M+i a \mu}{ \pm \sigma-M-i a \mu} \tilde{r}_{ \pm}, \quad R_{ \pm}=-\frac{ \pm \sigma+M-i a \mu}{ \pm \sigma+M+i a \mu} \tilde{R}_{ \pm}, \\
\tilde{r}_{ \pm} & =\sqrt{\rho^{2}+\left(z-\frac{1}{2} R \pm \sigma\right)^{2}}, \quad \tilde{R}_{ \pm}=\sqrt{\rho^{2}+\left(z+\frac{1}{2} R \pm \sigma\right)^{2}},
\end{aligned}
$$

where $M$ is the mass of each Kerr constituent, $a$ the angular momentum per unit mass of the lower constituent and $R$ the coordinate distance between the centers of the sources; the horizon's half length has the form

$$
\sigma=\sqrt{M^{2}-a^{2} \mu}, \quad \mu \equiv \frac{R-2 M}{R+2 M},
$$

while the corresponding metric functions are defined by the expressions

$$
\begin{aligned}
f= & \frac{A \bar{A}-B \bar{B}}{(A+B)(\bar{A}+\bar{B})}, \quad e^{2 \gamma}=\frac{A \bar{A}-B \bar{B}}{K_{0} \tilde{R}_{+} \tilde{R}_{-} \tilde{r}_{+} \tilde{r}_{-}}, \quad \omega=-\frac{2 \operatorname{Im}[G(\bar{A}+\bar{B})]}{A \bar{A}-B \bar{B}} \\
G= & -z B+R \sigma\left[2 R\left(R_{-} r_{-}-R_{+} r_{+}\right)+4 \sigma\left(R_{+} R_{-}-r_{+} r_{-}\right)\right. \\
& \left.-\left(R^{2}-4 \sigma^{2}\right)\left(R_{+}-R_{-}-r_{+}+r_{-}\right)\right], \\
K_{0}= & 16 \sigma^{4} R^{4} / M^{4} .
\end{aligned}
$$

This solution represents the pure vacuum limit of both electrovac metrics considered in our paper.

\section{Acknowledgments}

This work was partially supported by Project 128761 from CONACyT of Mexico.

[1] C. J. Ramírez-Valdez, H. García-Compeán, and V. S. Manko, Thermodynamics of two aligned Kerr black holes, Phys. Rev. D 102, 024084 (2020). 
[2] R. P. Kerr, Gravitational Field of a Spinning Mass as an Example of Algebraically Special Metrics, Phys. Rev. Lett. 11, 237 (1963).

[3] M. Appels, R. Gregory, and D. Kubizňák, Black hole thermodynamics with conical defects, J. High Energy Phys. 05, 116 (2017).

[4] P. Krtouš and A. Zelnikov, Thermodynamics of two black holes, J. High Energy Phys. 02, $164(2020)$.

[5] E. Newman, E. Couch, K. Chinnapared, A. Exton, A. Prakash, and R. Torrence, Metric of a rotating charged mass, J. Math. Phys. 6, 918 (1965).

[6] V. S. Manko, J. Martín, and E. Ruiz, Metric of two arbitrary Kerr-Newman sources located on the symmetry axis, J. Math. Phys. 35, 6644 (1994).

[7] W. Israel, Line sources in general relativity, Phys. Rev. D 15, 935 (1977).

[8] N. Bretón and V. S. Manko, A binary system of 'antisymmetric' Kerr-Newman masses, Class. Quantum Grav. 12, 1969 (1995).

[9] V. S. Manko, R. I. Rabadán, and E. Ruiz, The Bretón-Manko equatorially antisymmetric binary configuration revisited, Class. Quantum Grav. 30, 145005 (2013).

[10] F. J. Ernst, V. S. Manko, and E. Ruiz, Equatorial symmetry/antisymmetry of stationary axisymmetric electrovac spacetimes: II, Class. Quantum Grav. 24, 2193 (2007).

[11] V. S. Manko, R. I. Rabadán, and J. D. Sanabria-Gómez, Stationary black diholes, Phys. Rev. D 89, 064049 (2014).

[12] L. Smarr, Mass formula for Kerr black holes, Phys. Rev. Lett. 30, 71 (1973).

[13] L. Parker, R. Ruffini, and D. Wilkins, Metric of two spinning charged sources in equilibrium, Phys. Rev. D 7, 2874 (1973).

[14] S. D. Majumdar, A class of exact solutions of Einstein's field equations, Phys. Rev. 72, 390 (1947).

[15] A. Papapetrou, A static solution of the equations of the gravitational field for an arbitrary charge distribution, Proc. Roy. Irish Acad. A 51, 191 (1947).

[16] G. W. Gibbons and S. W. Hawking, Action integrals and partition functions in quantum gravity, Phys. Rev. D 15, 2752 (1977).

[17] R. Bach and H. Weyl, Neue Lösungen der Einsteinschen Gravitationsgleichungen, Math. Zeits. 13, 134 (1922).

[18] M. S. Costa and M. J. Perry, Interacting black holes, Nucl. Phys. B 591, 469 (2000). 
[19] N. Bretón, V. S. Manko, and J. Aguilar-Sánchez, On the equilibrium of charged masses in general relativity: the electrostatic case, Class. Quantum Grav. 15, 3071 (1998).

[20] V. S. Manko, Double-Reissner-Nordström solution and the interaction force between two spherical charged masses in general relativity, Phys. Rev. D 76, 124032 (2007).

[21] F. J. Ernst, New formulation of the axially symmetric gravitational field problem, Phys. Rev. 167, 1175 (1968).

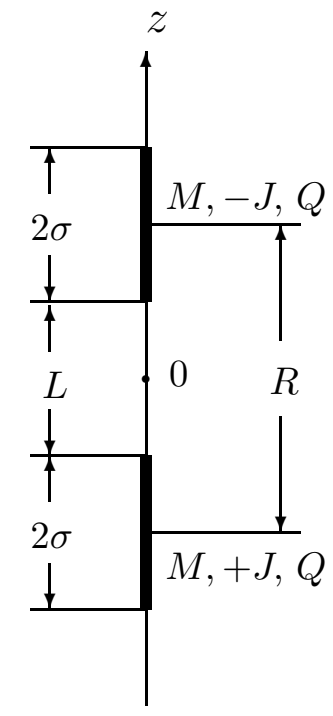

FIG. 1: Location of two equal counterrotating $\mathrm{KN}$ black holes with the same charges on the symmetry axis. 


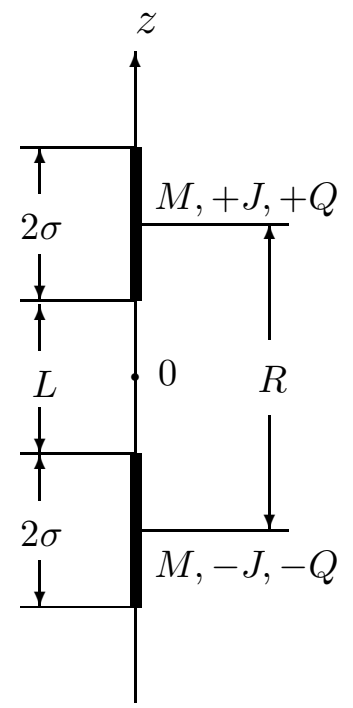

FIG. 2: Location of two equal counterrotating KN black holes with opposite charges on the symmetry axis. 